\title{
The Re-establishment of Synaptic Transmission by Regenerating Optic Axons in Goldfish: Time Course and Effects of Blocking Activity by Intraocular Injection of Tetrodotoxin
}

\author{
J. T. SCHMIDT, D. L. EDWARDS* and C. STUERMER
}

\begin{abstract}
(J.T.S.) Dept. of Biological Sciences, State Univ. of New York at Albany, 1400 Washington Ave, Albany, NY 12222, (D.L.E.) Dept. of Physiology, Cornell Univ. Medical College, New York, NY 10021, and (C.S.) Div. of Biological Sciences, Univ. of Michigan, Ann Arbor, MI 48109 (U.S.A.)
\end{abstract}

(Accepted November 16th, 1982)

\begin{abstract}
Key words: synaptic transmission - nerve regeneration - axonal transport - goldfish optic nerve - tetrodotoxin
\end{abstract}
\begin{abstract}
Intraocular injections of tetrodotoxin were used to block activity for 27 days in normal fish and for the first 27 or 31 days of regeneration in fish with one optic nerve crushed. Synaptic activity was then assessed by a current source-density analysis of field potentials evoked by optic nerve shock at different times following the TTX treatment. In normal fish, the lack of activity for 4 weeks had no significant effect on the maintenance of synaptic strength. Likewise, in fish with nerve crush, lack of activity did not prevent the regenerating optic fibers from forming synapses that were nearly as effective as those formed in controls injected with the citrate buffer vehicle. The earliest synapses were formed at the rostromedial corner of the tectum (where the tract enters) at 20 days after nerve crush, when fibers had not yet reached the caudal areas. By 28 days synaptic potentials could be recorded everywhere on the surface of the tectum in both controls and TTX injected fish. However, the latency of the responses with TTX were longer, suggesting a smaller caliber of fiber, which is consistent with an earlier finding of decreased axonal transport in TTX fish ${ }^{6}$. Maturation of the regenerating fibers proceeded slowly in both TTX and control fish. After more than 5 months, the projections were nearly normal but still not completely normal.
\end{abstract}

\section{INTRODUCTION}

The role of action potentials in the development of the nervous system is not very well understood. Both in vitro and in vivo preparations have been employed to assess the role of action potentials in development. Tissue or cell culture has furnished a well-controlled situation where activity can be eliminated entirely during synaptogenesis. Some experiments have employed tetrodotoxin(TTX) ${ }^{19}$ or xylocaine ${ }^{4}$ to eliminate spike activity and found that silent fibers still have the ability to form effective and morphologically normal synapses ${ }^{19}$. However, other studies have shown that motorneurons in TTX culture, even though they are able to form connections with muscle fibers, become shrunken and lose much of their choline acetyltransferase activity'.
Among the in vivo preparations, those involving sensory deprivation are most common ${ }^{11,12}$. However, while deprivation may lower the level of activity, it does not abolish activity entirely, and hence the interpretation of these experiments in this regard is not clear. Recently, one in vivo experiment has clearly eliminated action potentials during development of the eye and its projection. Harris ${ }^{9}$ implanted an axolotl eye into a host newt which produces TTX. Although he was able to show that the eye and its retinal ganglion cells survived and made morphologically normal synapses within the newt tectum, several questions remained unanswered because he was unable to reverse the TTX block and record electrophysiologically. Two of these questions are (1) whether the synapses formed by the silent fibers are as effective as those formed by active ones, and (2) whether the projection formed by

\footnotetext{
* Present address: Dept. Neurobiology, Harvard Medical School, 25 Shattuck St., Boston, MA 021 15, U.S.A.
} 
the silent eye has the normal degree of retinotopic order. To answer these questions, we have used repeated intraocular injections of TTX during optic nerve regeneration in goldfish to produce a continuous block of activity. Since this block is reversible, we could employ the current source-density analysis ${ }^{24}$ to show electrophysiologically that the synapses formed are nearly as effective as those of controls. In the following paper $^{26}$ we employ unit recordings to show that activity is necessary for the fine tuning of the retinotopic order.

\section{MATERIALS AND METHODS}

Goldfish, $10-13 \mathrm{~cm}$ in overall length were purchased from Ozark fisheries (Stoutland, MO) and kept at approximately $20^{\circ} \mathrm{C}$, except for one small group which was maintained at $25^{\circ} \mathrm{C}$. Before surgery or recording, fish were anaesthetized by immersion in a $0.1 \%$ solution of tricaine methanesulfonate. The right optic nerve was exposed and crushed in the orbit using fine forceps under visual control, leaving the sheath intact.

\section{TTX injections}

The eye was injected just behind the iris with either a solution containing $70 \mathrm{ng}$ of tetrodotoxin and $350 \mathrm{ng}$ of citrate or a control solution containing only the citrate. The injections were made under semisterile conditions, in order to minimize potential problems with inflammation of the eye or with formation of cataracts in the lens or cornea. Repeated injections were made through the same puncture in the sclera. The resulting concentration in the vitreous of the eye was calculated to be approximately $4 \mu \mathrm{M}$ for TTX and $20 \mu \mathrm{M}$ for citrate. The block of neural activity was assessed behaviorally (see Edwards and Grafstein ${ }^{6}$ or electrophysiologically (tectal recording), and the dose was determined in the following way. A threshold dose was first determined to be approximately $0.3 \mathrm{ng}$. Ten times this dose blocked activity for $1-1.5$ days. Increasing the dose to 100 times threshold blocked activity for 2-2.5 days, affording only one extra day of block, and large single doses of $1 \mu \mathrm{g}$ or more were often fatal for the fish. We therefore used repeated smaller injections which seemed to have no noticeable extraocular toxic effects. The TTX apparently did not accumulate outside the eye since the blocked eyes made normal eye movements, indicating that the sodium channels in both the oculomotor nerves and the extraocular muscles remained largely unblocked.

\section{Experimental groups}

In two groups of fish the optic nerve was crushed and a block of neural activity was maintained with repeated injections of TTX. The first group (TTX-constant), received injections every 2.5 days following nerve crush. This schedule produces a continuous block of activity. The second TTX group (TTX-tested) received injections at 4-day intervals, allowing the block to wear off for 18-24 h to allow behavioral testing, the results of which are reported in a separate study ${ }^{6}$. Since no differences were observed between the TTX-constant and TTX-tested fish, they will often be referred to jointly as TTX-regen fish.

\section{Control groups}

Three control groups were used. One control regeneration group (Citrate-regen) received injections of the citrate buffer on the same schedule as the TTX fish. A second control group regenerated without any injections. These were used to establish the earliest feasible time for recording. A third control group (TTX-normal) did not have their nerves crushed but received TTX injections every 2.5 days for a continuous block of 27 days.

\section{Histology}

In order to visualize anatomically the regenerated fibers, $10 \mu \mathrm{l}$ of a $30 \%$ solution of horseradish peroxidase (HRP, Miles) was injected into the eye either one or two days before recording and sacrifice. Fish were perfused with Ringer's solution followed by a mixture of $1 \%$ paraformaldehyde and $2 \%$ glutaraldehyde. The brains were postfixed for $8 \mathrm{~h}$, transferred to a phosphate buffer solution, cut into $100 \mu \mathrm{m}$ sections and reacted with tetramethyl benzidine according to the 
method of Mesulam ${ }^{14}$. Camera lucida drawings were made of the extent of labelling in these sections. An electron microscopic study of the regenerating optic fibers will appear elsewhere ${ }^{29}$.

\section{Electrophysiology}

A current source-density analysis of field potentials evoked by optic nerve shock was undertaken from 4 to 137 days after the last TTX or control injection. Fish were carefully checked before recording and those few with any indication of abnormality of the eye were discarded. Later histological examination of similar retinae by Edwards and Grafstein ${ }^{6}$ showed no abnormalities of the retina, including no loss or shrinkage of ganglion cells. The recording methods as well as the numerical methods for data analysis have been described in great detail elsewhere ${ }^{24}$. Briefly, the eye was removed, a concentric bipolar suction electrode was placed over the stump of the optic nerve and electric shocks of short duration were administered at low frequency from an optically isolated stimulator. The field potentials were recorded (DC) in the tectum with a Ringer-filled pipette, relative to a silver-chloride ground electrode behind the cerebellum. Analogue-to-digital conversions, the averaging of traces and the second differencing calculations of the sources and sinks of synaptic currents were performed with an LSI-11/03 based microcomputer.

\section{RESULTS}

\section{Field potential analysis of normal goldfish retino-} tectal projection

The optic nerve of the normal goldfish contains 3 classes of optic nerve fibers as determined by conduction velocity ${ }^{24}$. These groups of fibers project to characteristic laminae within the tectum. For comparison with later groups of fish, the recording site was standardized to a position just caudal of the center of the tectum viewed from above. One full set of records from a normal fish is shown in Fig. 1. Approximately 3-4 ms after the stimulus, the first optic fiber volley (marked by a dot in traces) arrived at the tectum, reflecting a conduction velocity of $4-5 \mathrm{~m} / \mathrm{s}$.
At superficial levels the first fiber volley was followed by a broad negativity peaking at approximately $8 \mathrm{~ms}$. This broad negativity was actually an aggregate of two postsynaptic responses slightly separated in time. This can be demonstrated clearly at $200-250 \mu \mathrm{m}$ where the earlier component becomes smaller and begins to reverse while the second is still very large and near its maximum. Deeper, at $300-400 \mu \mathrm{m}$, both have inverted to become positive. This inversion occurs because most tectal cells receiving superficial optic input are vertically oriented and their activation makes them become current dipoles ${ }^{24}$. The sources and sinks (on the right), calculated by the second differencing method, more accurately localize the depths of these two components. P1 occurs primarily at $100 \mu \mathrm{m}$, whereas P2 occurs primarily at $200 \mu \mathrm{m}$. Both of these sinks (excitatory synapses) occur within the superficial gray and white layer (SFGS), the primary optic termination zone which contains two plexiform layers ${ }^{13}$. In addition to the differences in depth for components $\mathrm{Pl}$ and $\mathrm{P} 2$, it is possible at $200-300 \mu \mathrm{m}$ to see a second presynaptic deflection riding on the field potentials. This deflection corresponds to the second optic fiber volley. Finally there is a third, longer latency, broad postsynaptic deflection in the deeper layers of the tectum at $250-400 \mu \mathrm{m}$. This has been associated with a slower conducting optic fiber group projecting to the middle of the central gray layer and to the deep white layer ${ }^{24}$. The source-sink records show sinks of synaptic current there also.

\section{The re-establishment of synaptic transmission fol- lowing regeneration}

Since the re-establishment of synaptic transmission after nerve crush has not yet been studied in the goldfish retinotectal system, we have first established the normal time course of its return for comparison with fish regenerating during TTX block. Recordings were made from 40 fish over a time span from 14 to 161 days after nerve crush. Of these, 15 had received TTX injections, 14 had received citrate injections and 11 had received no injections during regeneration. Qualitatively, the field potentials of the 

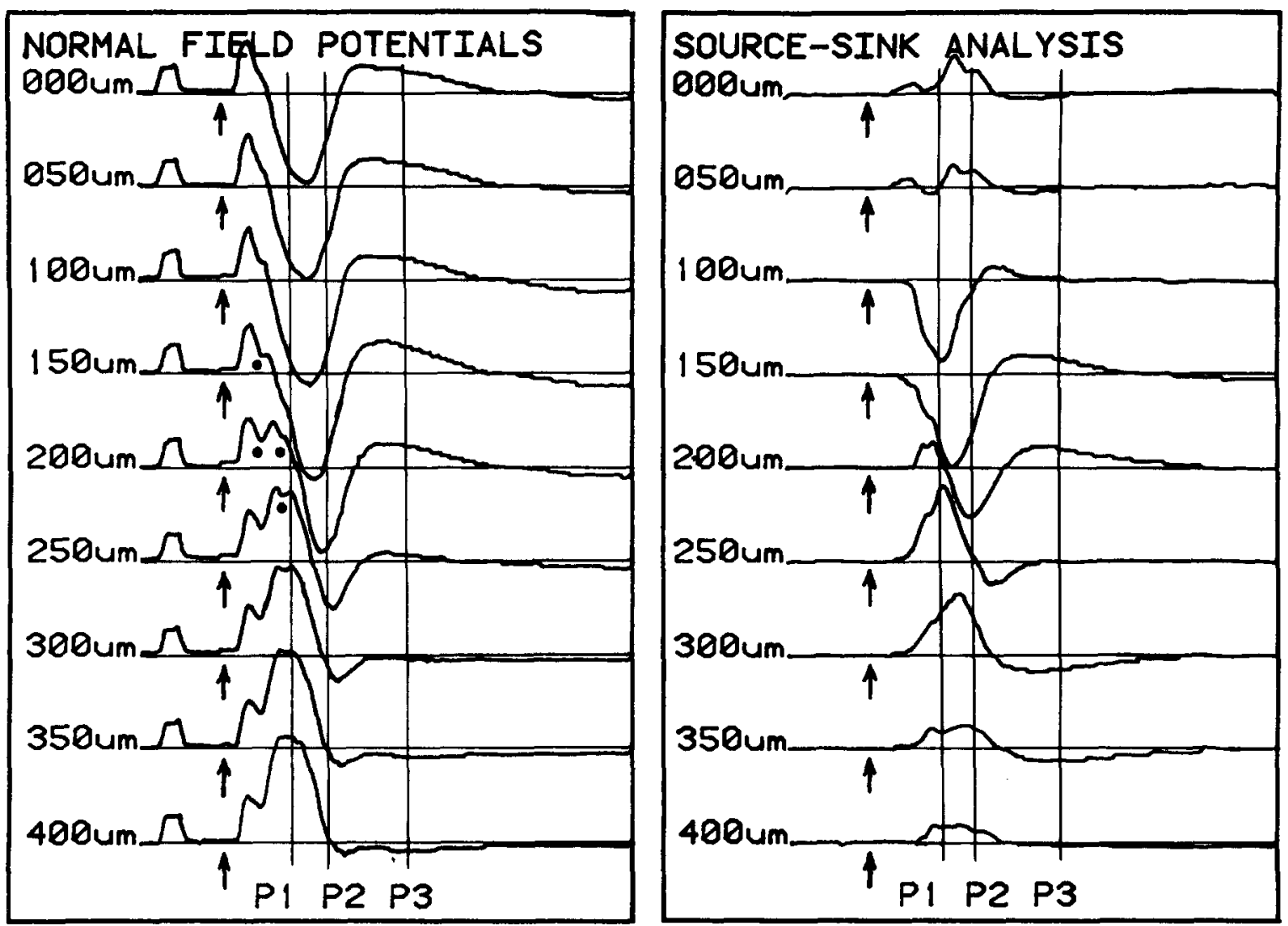

Fig. 1. A set of traces from a normal fish. The field potentials were elicited by optic nerve shock (arrows); the artifact was electronically suppressed. Depths are shown next to each trace which is actually an average of 10 traces. The vertical lines mark the 3 postsynaptic components (P1, P2 and P3). Two of the presynaptic deflections are indicated with dots. Negative is downard. Trace length is $40 \mathrm{~ms}$. Calibration pulse: $2 \mathrm{~ms} \times 1 \mathrm{mV}$. On the right are the sources and sinks of synaptic current computed by the second difference technique. Sinks are downward deflections.

TTX-blocked fish did not differ from controls so that they will be considered with the control groups in establishing the time course.

Early regeneration-Spatiotemporal pattern of synapse formation. One important question about regeneration is whether the first arriving fibers immediately make synapses at their point of entry ${ }^{29}$ or whether they wait until the full complement of fibers has arrived before making effective connections as has been suggested for the developing chick retinotectal system ${ }^{5}$. Since the route of the fiber tracts leading into the tectum is well established, the pattern may be inferred from individual fish by comparing responses from a number of tectal positions at very early stages in regeneration. The spatial pattern of responses likewise may be compared with the pro- gress of the regenerating fibers assessed by anterograde transport of HRP.

At very early times, the fine regenerated fibers fatigued extremely rapidly. Consistent responses could be obtained only at a rate of about one stimulus per $10 \mathrm{~min}$, which made recording very time consuming and averaging very difficult. Table I shows the results for the 11 fish which were recorded at or before 20 days. Representative traces from superficial tectum are shown in Fig. 2. In the fish kept at $20^{\circ} \mathrm{C}$, no responses could be obtained at 18 and 19 days. For these fish the first response was obtained at 20 days. Likewise in the fish kept at $25^{\circ} \mathrm{C}$, no responses could be elicited at 14 and 16 days, and responses were first obtained at 18 days. In both cases the earliest responses were restricted to the extreme rostromedial corner where the tract en- 


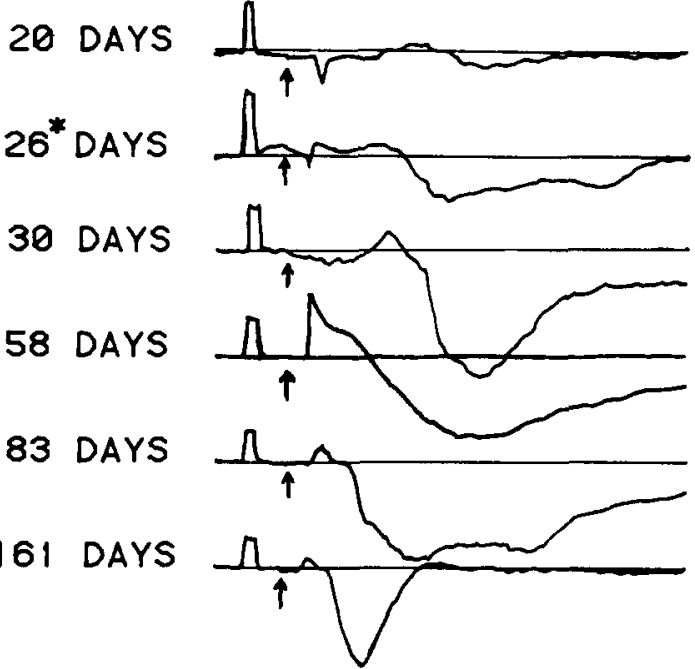

Fig. 2. Representative traces recorded from superficial tectum (150 $\mu \mathrm{m}$ depth) at various times after optic nerve crush. Asterisk indicates a fish kept at $25^{\circ} \mathrm{C}$, although the time has been corrected to the equivalent at $20^{\circ} \mathrm{C}^{27}$. Calibration pulses: $2 \mathrm{~ms} \times 0.5 \mathrm{mV}$ for the upper 4 traces, $\times 1 \mathrm{mV}$ for the lower 2 traces. Trace length is $80 \mathrm{~ms}$.

ters. Thus the nerves of fish kept at $25^{\circ} \mathrm{C}$ tended to regenerate faster as found previously in behavioral studies ${ }^{27}$. At 19 days in the $25^{\circ} \mathrm{C}$ fish, responses could be recorded all over the tectal surface, but the responses were graded in amplitude from rostral to caudal (Fig. 3). In addition, there was a noticeable gradient from medial periphery towards central tectum at rostral levels. Such gradients might be predicted from the path of entry of the fibers and their spatiotemporal spread across the tectum as seen by radioautographic labelling ${ }^{29}$ or in our HRP fills in these same fish (Fig. 3).

The HRP fills demonstrated optic nerve fibers in the tectum at times before the field potentials could be recorded ( 14 and 16 days at $25^{\circ} \mathrm{C}$ and 18 and 19 days at $20^{\circ} \mathrm{C}$; see Fig. 3 and Table I). In the two cases where responses were present only rostromedially, the HRP showed the fibers covering somewhat larger areas of the tectum,

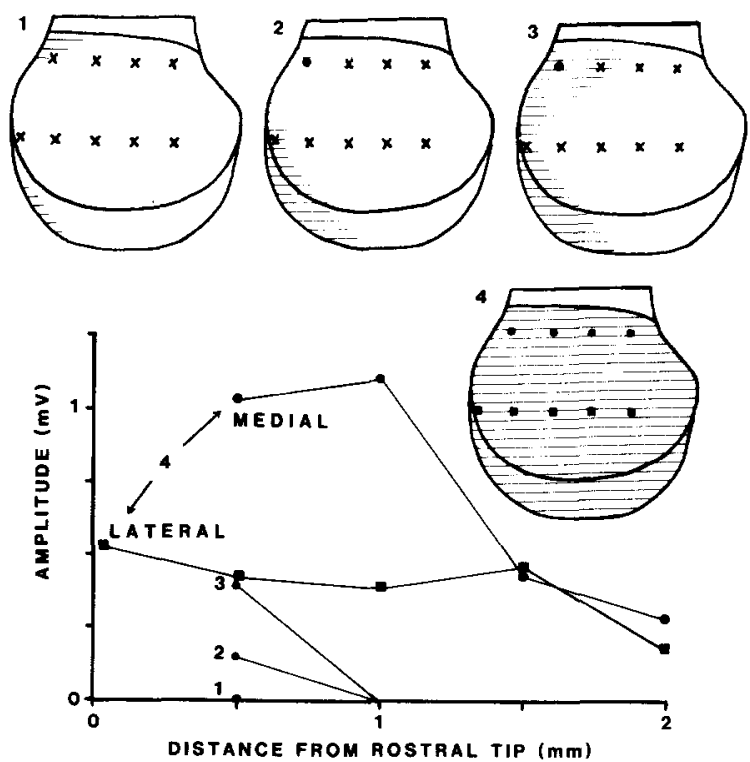

Fig. 3. A plot of the amplitude of the superficial negative responses (such as those in Fig. 2) vs rostrocaudal position on the tectum. The data are taken from the 8 fish of Table I. The recording positions are marked on the outlines of the tectal surface as seen from above. Circles indicate a medial series of penetrations, squares a lateral series, and x's the positions where no responses could be recorded. The 4 tectal outlines above the plot show the extent of the HRP labelling both on the dorsal surface and in the portion which curves under laterally. 1 , four fish, at 14-16 days at $25^{\circ} \mathrm{C}$ and $18-19$ days at $20^{\circ} \mathrm{C}$, no responses; 2 and 3 , two fish which each gave responses only at the extreme rostromedial corner, 20 days at $20^{\circ} \mathrm{C}$ and 18 days at $25^{\circ} \mathrm{C}$, respectively; 4 , a 19-day fish at $25^{\circ} \mathrm{C}$ which gave potentials all over the tectal surface.

\section{TABLE I}

\section{Summary of results in early regeneration period}

\begin{tabular}{llll}
\hline $\begin{array}{l}\text { Days since } \\
\text { nerve crush }\end{array}$ & $\begin{array}{l}\text { Temp } \\
(C)\end{array}$ & Field potls & HRP labeling \\
\hline 14 & 25 & No resp. & Tracts labeled rostrally \\
16 & 25 & No resp. & Tracts labeled rostrally \\
18 & 25 & RM corner only & Label heavy in tracts, light over terminal zone rostrally \\
19 & 25 & All over tectum & No fill attempted \\
26 & 25 & No recording & Entire tectum labeled \\
18 & 20 & No resp. & Tracts labeled rostrally \\
19 & 20 & No resp. & Tracts labeled rostrally \\
20 & 20 & RM corner only & Tracts heavily labeled to midtectum, light label over terminal zone \\
& & & rostrally \\
\hline
\end{tabular}


although not completely filling it (Fig. 3). Mostly this was attributable to the fact that much of the HRP reaction product in these early cases was in the tracts and in stratum opticum, the extension of the optic tracts, which are separate from the synaptic terminal zones in the tectum. Also the limit of detection for the field potentials was about $100 \mu \mathrm{V}$ when only a few traces were averaged together. Thus some connections may have been formed before they could be detected, although these could not have been numerous. Nevertheless, comparing the field potential data with the HRP data clearly shows that effective synapses are indeed made rostrally before the fibers arrive in the caudal and central areas.

Time course of regeneration and the effects of $T T X$. By 28 days in the $20^{\circ} \mathrm{C}$ fish, responses could always be recorded at any point on the tectal surface. Because of this and the relevance of this time to the return of behavior (see discussion), extensive recordings and comparisons between TTX fish and controls were begun at this point. Even at 28 days, the responses although consistent, still fatigued extremely rapidly. At most, 3 responses could be elicited every $10 \mathrm{~min}$. The amplitude of the response was always small (less than $1 \mathrm{mV}$ ), the latency was of-

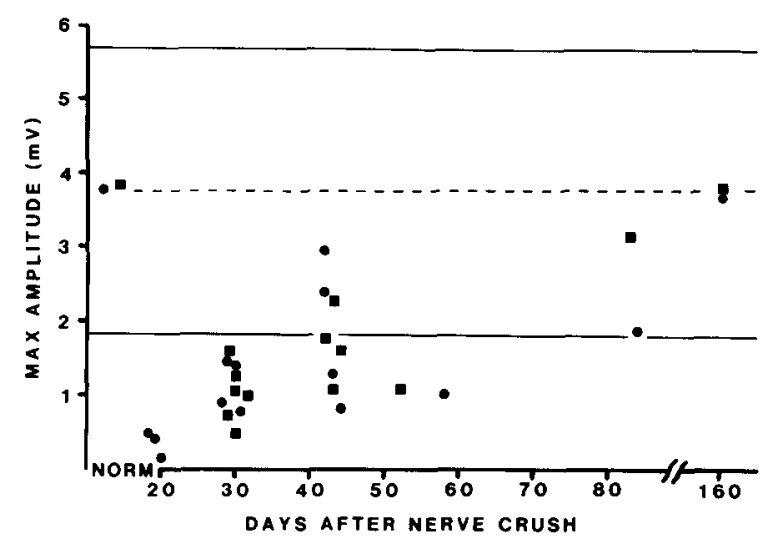

Fig. 4. A plot of the amplitude of the superficial negative field potentials vs time after nerve crush. Squares indicate fish injected with TTX, circles the citrate injected controls. Each point represents one regenerated fish exept the two points at the place marked 'normal'. Those are the means of the normals and TTX-injected normals. The dashed line and solid lines are the mean and two standard deviations around the mean of the normal group.

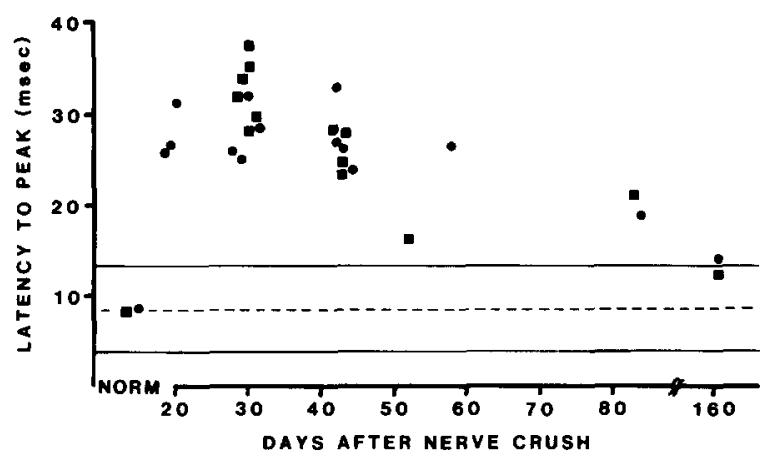

Fig. 5. A plot of the latency to the peak of the superficial negative response vs time after nerve crush. Circles, squares and lines as in Fig. 4.

ten over $30 \mathrm{~ms}$ (refelcting the fine unmyelinated fibers), and the response had an extended duration. These 3 aspects were quantified and they are plotted in Figs. 4, 5 and 6. Fig. 4 shows that the amplitude of the responses rose with time from less than $0.5 \mathrm{mV}$ at 20 days, reaching mean levels for the normal fish (almost $4 \mathrm{mV}$ ) by 161 days. Two other points are clear from Fig. 4. First, there was a great deal of variability in the responses from fish at the same postcrush interval. This would appear to reflect an inherent variability in the regeneration process, which persists for months. Second, there was no detectable difference in the amplitude of the responses of TTX and citrate fish, either early or late in regeneration (Two tailed $t$-test). Likewise, there was no difference between TTX fish blocked constantly (2.5-day interval) versus those allowed to regain vision briefly for behavioral testing (4-day interval). Thus there appears to be no dependence of the amplitude on the activity in the optic fibers during regeneration.

The latencies to peak response in superficial tectum are plotted in Fig. 5. Whereas the normal latency to peak is $8 \mathrm{~ms}$, at early times the latency averages nearly 4 times that value. Assuming a direct path length of $10 \mathrm{~mm}$ to the standard recording site just caudal to the midtectal region, this corresponds to a conduction velocity of approximately $0.2-0.4 \mathrm{~m} / \mathrm{s}$. The latency was shorter by 85 days (reflecting myelination), but only reached the $95 \%$ confidence interval of the normals by 161 days.

Early in regeneration, the TTX treatment ap- 
peared to have an effect upon the latency of the responses. On the average, there is a significant tendency for the TTX fish in the 28-31 day range to have longer latencies than the controls recorded at the same time ( $P<0.05$, two-tailed $t$-test). On the other hand, the responses of TTX fish at 42-84 days were not significantly slower, and in fact have slightly shorter latencies on the average than controls. Since fish recorded from 42 days onward recovered from TTX block on day 31 , they had a longer interval with activity before recording than those which recovered at 27 days and were recorded within 2-4 days thereafter. Thus the TTX block tends to produce longer latency responses, a difference which later disappears upon restoration of activity.

The widths of the responses at half maximum amplitude, which we used as a convenient measure of response duration, are plotted in Fig. 6. Even more than the other plots, Fig. 6 shows extreme variability which can be seen to persist for months. The width drops within the normal range only at 161 days, and thus is probably also correlated with advanced myelination. The extremely high values, especially those at 83-84 days, reflect the appearance of a double peak in the superficial negative response (see Fig. 2). The persistence of the long durations at 83-84 days contrasts with the decreased latency at those times (Fig. 4). It appears to reflect the persistence of the long latency response while the main peak has moved to shorter latencies as myelination proceeds. Examination of the plot reveals a tendency for the TTX fish to have longer

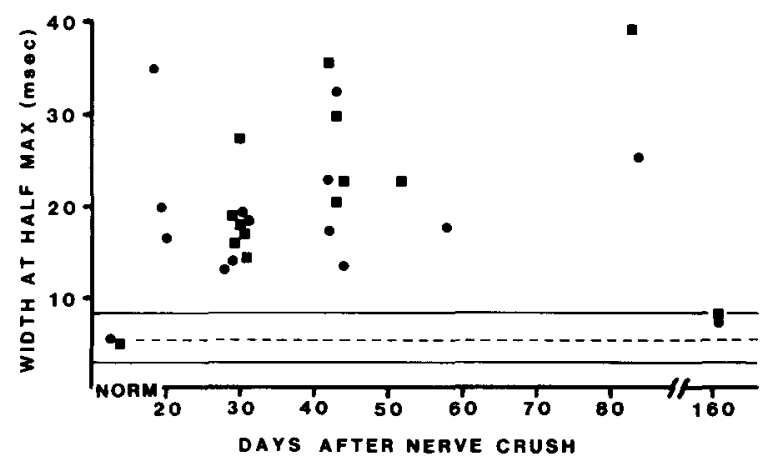

Fig. 6. A plot of the width of the superficial negative response at half its maximum vs time after crush. Circles, squares and lines as in Fig. 4. durations. However, statistically this tendency is not significant $(P=0.12$, two-tailed $t$-test).

The source-sink analysis of both a citrate and a TTX-regenerate fish are shown in Figs. 7 and 8. The field potentials of Fig. 7 which were recorded from a citrate control fish at 42 days after nerve crush show a case of orderly regeneration. The same 3 conduction velocity groups as in the normal (Fig. 1) can be seen, although they occur at longer latencies (note that the trace length here is twice that in Fig. 1). The 3 groups of fibers are also associated with the correct depths. Although sinks were never found significantly outside the usual laminae for the optic projection, occasionally the conduction velocity groups would be arranged inappropriately within those laminae, something never seen in normal fish. Thus the fastest conducting group (P1) would sometimes appear deep rather than superficial to the second group (P2). Interchanging of fibers between groups $\mathrm{P} 2$ and $\mathrm{P} 3$ or $\mathrm{P} 1$ and $\mathrm{P} 3$ was also common. Fig. 8 shows such effects in a TTX-tested fish, but tabulation of all the results showed that it did not occur significantly more often in the TTX fish than in the citrates. Thus it would appear to be due to the inherent variability of the regeneration process itself, rather than a deficit due to the lack of activity.

Fig. 9 shows the full set of records from one of the long-term (161 day) fish, and demonstrates that for the most part these errors appear to be rectified with time as both the fish recorded at this time showed nearly normal lamination of the 3 classes of input. The slight sink that persists at long latency in the $150 \mu \mathrm{m}$ trace is most probably a group of yet-to-be myelinated fibers rather than a misdirected group of $\mathrm{P} 3$ fibers as these occur at shorter latencies. In addition this long latency sink at $150 \mu \mathrm{m}$ often occurs in normal fish at tectal positions near the periphery where the yet-to-be myelinated fibers are known to project.

\section{Effect of blocking activity on the maintenance of synaptic connections}

Fish in the TTX-normal group had a continuous 27-day block of spike activity in the optic nerve fibers to test whether activity is necessary 

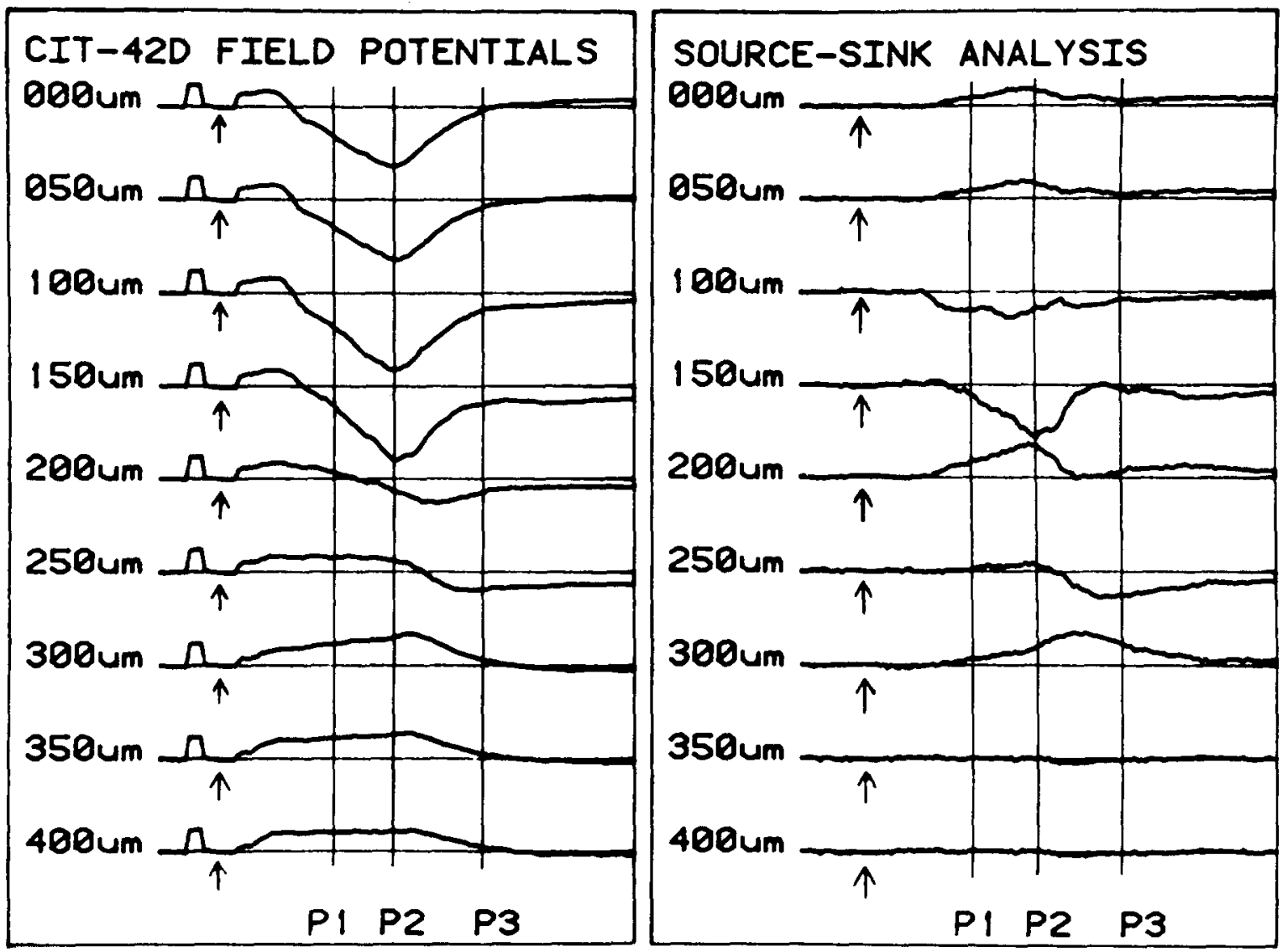

Fig. 7. A set of traces recorded 42 days after nerve crush in a fish injected with citrate buffer. Trace length is $80 \mathrm{~ms}$. Calibration pulse is $1 \mathrm{mV} \times 2 \mathrm{~ms}$. The 3 postsynaptic components are marked with vertical lines. Other conventions as in Fig. 1.

to maintain the efficacy of their synaptic transmission. These fish received their final TTX injection on day 24 and were recorded on day 28 , within $24 \mathrm{~h}$ of the release from the block of action potentials. The field potentials recorded from all 3 of these fish were similar in nearly all respects to those from normal fish. One full set of records from a TTX-normal fish is shown in Fig. 10. All 3 synaptic components could be seen in the TTX-normal fish. In order to make quantitative comparisons, measurements were made, as before, of the latency, of the maximum amplitude of the negative deflection, and finally of its width at half its maximum. The measurements for these fish are listed in Table II along with the means for a control group of 10 normal fish. There was no tendency for the TTX-normal fish to have field potentials that were smaller, or longer latency or longer duration than normal. In all cases $t$-tests showed the differences to be insignificant. These data indicate no loss of synaptic efficacy during the 27 days that spike activity was blocked.

Two minor differences were noted. First, the voltages necessary for maximal stimulation of the nerve in TTX-normal fish were consistently several times greater than for normal fish. Second, repeated stimulation of 1 per $10 \mathrm{~s}$ (with strength set at twice the value for maximal response) led over several minutes to a diminished response in these fish. This was never seen in normals. The response could be restored to full amplitude, however, by increasing the stimulus strength, indicating that the cause was axonal rather than synaptic fatigue. A more systematic study was accomplished by measuring the maximum response of the superficial negative wave at higher stimulus frequencies. The response decremented to $1 / 3$ at approximately $3-5 / \mathrm{s}$ in the TTX-normal fish versus $50 / \mathrm{s}$ for normals. In 

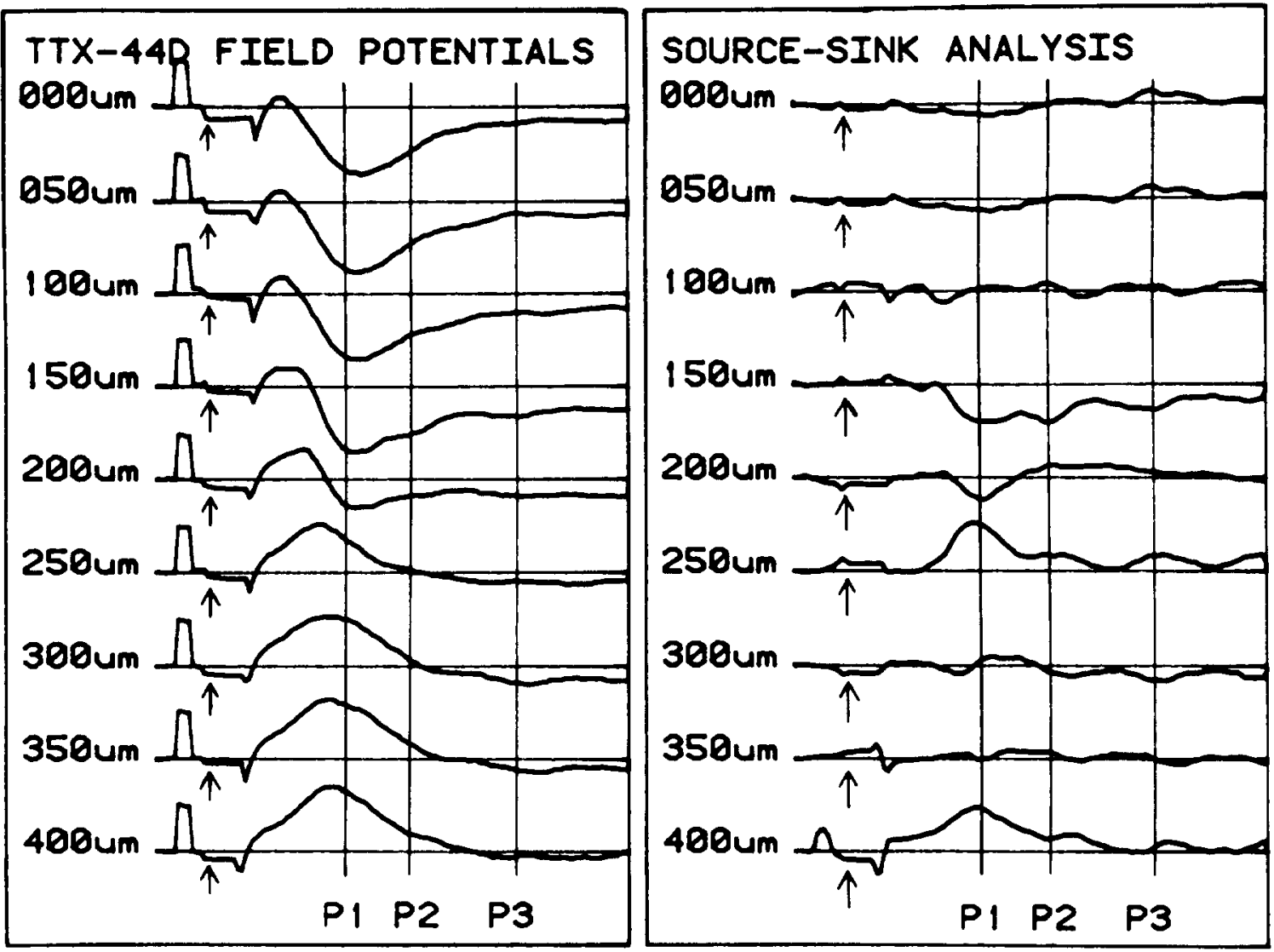

Fig. 8. A set of traces recorded 44 days after nerve crush in a fish injected with TTX from 0 to 31 days. Conventions as in Fig. 7.
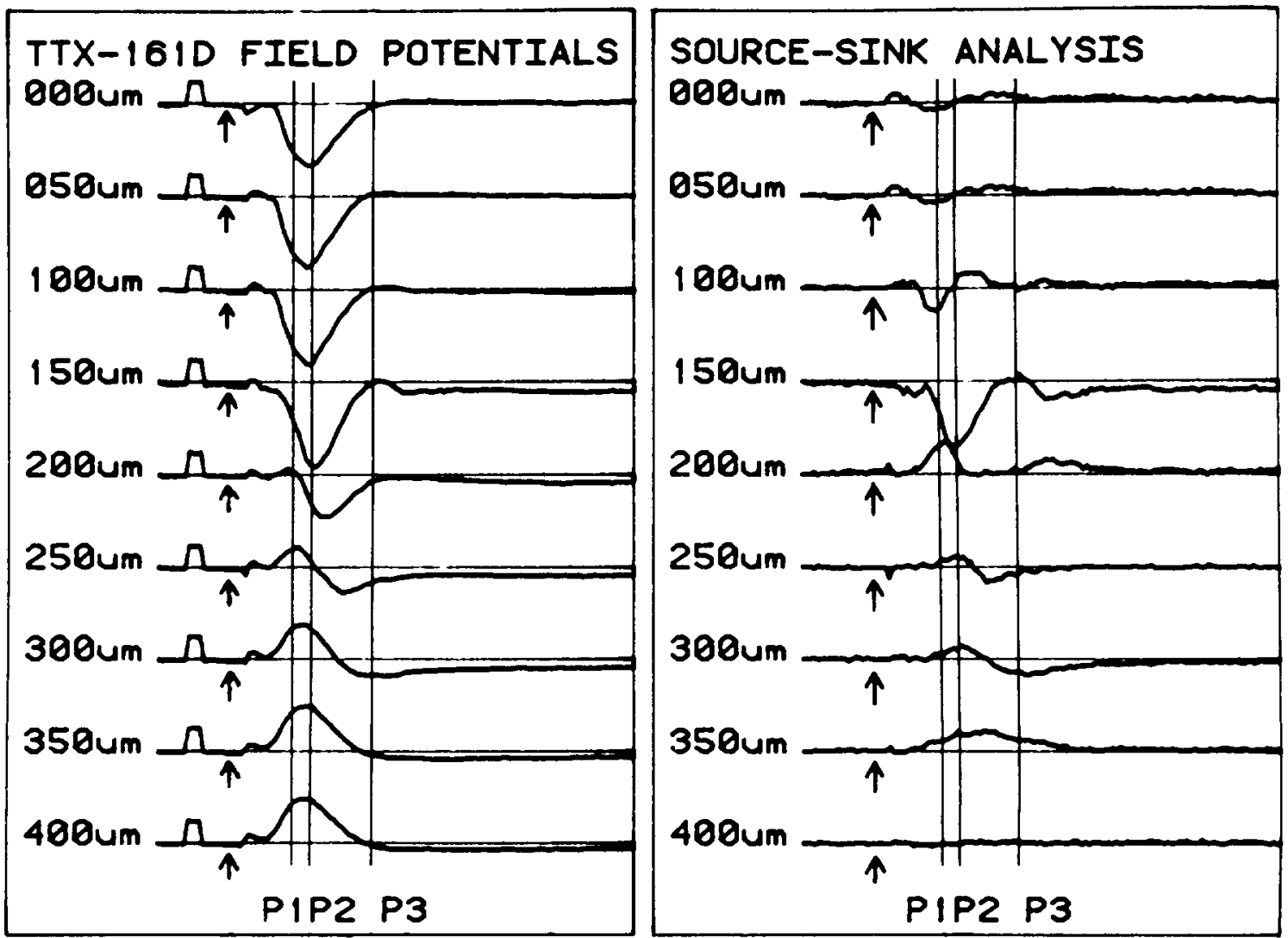

Fig. 9. A set of traces recorded 161 days after nerve crush in a fish injected with TTX from 0 to 31 days. Conventions as in Fig. 7. 

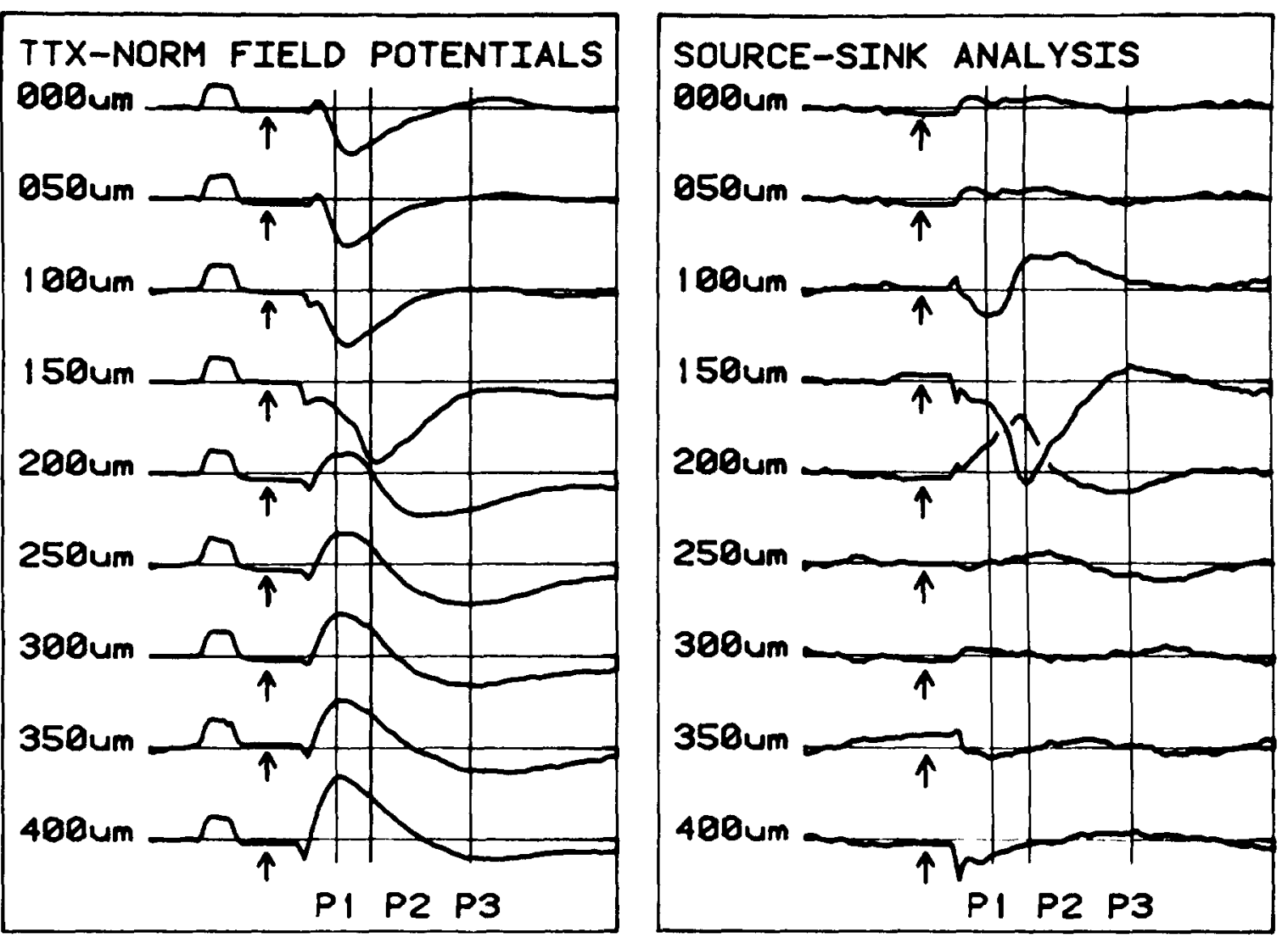

Fig. 10. A set of traces from a fish which had just recovered from a 27 -day block of activity without nerve crush. Trace length is 40 $\mathrm{ms}$. Calibration pulse: $1 \mathrm{mV} \times 2 \mathrm{~ms}$. Vertical lines mark the 3 postsynaptic components which were the same as in the normal (Fig. 1). Conventions as in Fig. 1.

normals the decrement is totally due to synaptic fatigue as the size of the presynaptic deflection remains constan $\mathrm{t}^{24}$, whereas for the TTX-normal fish, the postsynaptic decrement is accompanied by a marked decrement in the presynaptic deflection.

\section{DISCUSSION}

The main conclusion of this study is that the

TABLE II

Response measurements of TTX-normal fish

\begin{tabular}{llll}
\hline Fish/Group & $\begin{array}{l}\text { Latencyto } \\
\text { peak }(\mathrm{ms})\end{array}$ & $\begin{array}{l}\text { Amplitude } \\
(\mathrm{mV})\end{array}$ & $\begin{array}{l}\text { Width at half } \\
\text { maximum }(\mathrm{ms})\end{array}$ \\
\hline 28D TTX-norm & 7.25 & 6.13 & 4.62 \\
28D TTX-norm & 9.41 & 1.88 & 5.09 \\
28D TTX-norm & 8.00 & 3.55 & 5.49 \\
Mean TTX-norm & $8.22($ S.D. $=1.1)$ & $3.85($ S.D. $=2.14)$ & $5.07($ S.D. $=0.44)$ \\
Normals $(\mathrm{n}=10)$ & $8.58($ S.D. $=2.33)$ & $3.77($ S.D. $=0.97)$ & $5.61($ S.D. $=1.39)$ \\
\hline
\end{tabular}

absence of neural activity has no effect upon the maintenance of normal retinotectal synaptic efficacy and only a very slight effect upon the formation of effective connections during regeneration. In addition these experiments have provided much information about the time course of the maturation of newly regenerated projections. 


\section{Normal fish treated with TTX}

Results obtained from these animals demonstrated that the intact synapses maintain their efficacy when silenced for 4 weeks. Similar TTX blocks of motor nerves in rats also do not cause any loss of synaptic efficacy, but they do cause a sprouting of the fibers (including the silenced fibers) at the neuromuscular junction ${ }^{2}$. This sprouting is thought to be the result of the spread of acetylcholine receptors (AChR's) across the muscle fiber's surface ${ }^{22}$. The retinotectal projection of the goldfish is also thought to be nicotinic cholinergic ${ }^{720,21,23,25}$. In the goldfish tectum, neither sprouting nor extrasynaptic proliferation of AChR's has been assessed. The present results would appear to argue against any massive sprouting and AChR proliferation response, since there was no tendency for an increased amplitude of response after the 27 days of inactivity.

The decreased excitability of the fibers and the axonal fatigue at low frequencies are probably the direct effect of lingering TTX. Although the TTX was injected intraocularly, it is reasonable to assume that enough of it may have diffused into the optic nerve to block a small fraction of the sodium channels at the site of stimulation which was approximately $3 \mathrm{~mm}$ from the eye. The blockage of a small fraction of sites would hold the axons in a state of relative refractoriness. The blocked sodium channels would not be available for activation because of the TTX binding, just as a substantial fraction is not available because of inactivation ( $h$ factor) during the relative refractory period after each action potential ${ }^{10}$. Such a partial blockage of sodium channels could also produce a slowing of conduction velocity, but none was evident here. This would seem to indicate that the TTX diffused only a short distance along the nerve to the stimulating site but not far enough beyond to slow conduction.

\section{Time course of normal regeneration}

Postsynaptic responses of fibers regenerating at $20^{\circ} \mathrm{C}$ were first recorded at 20 days. This is some 6 days after the first detection of fibers reaching the tectum via axonal transport of HRP or labelled protein ${ }^{6,17}$. This lag is partially attributable to the presence of fibers within tectal tracts before terminals are formed within synaptic laminae, and also partially to a delay in the establishment of sufficient synaptic density for electrophysiological detection. At $25^{\circ} \mathrm{C}$ our first positive recordings were at 18 days while the first synapses were seen only one day earlier in an electronmicroscopic study ${ }^{29}$. Thus, it is clear from these recordings and the electromicroscopic evidence ${ }^{29}$ that effective synapses form rostrally before the optic fibers have reached caudal levels. From these experiments we do not know whether the fibers which form these early synapses are from inappropriate retinal areas, but other anatomical studies have shown that retinal fibers initially deploy very widely in the tectum ${ }^{8,15,28}$, and this suggests the need for a mechanism other than surface recognition to produce the refinement of the retinotopic map. The paper which follows ${ }^{26}$ presents evidence that this second mechanism is an activity dependent stabilization of those synapses which were formed in the correct area and retraction of other inappropriate ones.

After nerve crush, the field potentials took suprisingly long to return to normal. Even at 83-84 days, some 50 days after the return of behavioral function ${ }^{6}$, the responses were still grossly abnormal in latency and duration, and also slightly smaller than normal in amplitude. Our measurements of the amplitude of the postsynaptic response may be compared with measurements of several synaptic components made by others. The amplitude recovers with a time course similar to that of AChR levels following nerve crush $^{23}$. These return to normal around 80 to 100 days. The drop in AChR levels following nerve crush or enucleation is thought to be due to the removal of the postsynaptic densities ${ }^{18}$. Two other indices of synaptic function, choline acetyltransferase and acetylcholinesterase, return to normal by 70 days'. Finally, density of synapses also takes at least 80-90 days to recover ${ }^{16}$.

The response latency and duration in the early stages of regeneration are likely to reflect somewhat different processes than those at later stages. At early stages, all the regenerating fibers 
are unmyelinated, and this results in a long latency response. The calculated conduction velocity of $0.2-0.4 \mathrm{~m} / \mathrm{s}$ is close to that of the unmyelinated optic fibers seen in the normal frog ${ }^{3}$. The prolonged duration is most likely due to variability both in the diameters of the axons and in their path lengths before making synapses. Electron micrographs clearly show a great deal of variability in the caliber of regenerating optic axons $^{16,31}$, and roundabout paths are common in single fibers filled following regeneration in newts ${ }^{8}$. Later in regeneration, the degree of myelination probably plays the greatest role in shaping the time course of the response. Myelination is known to begin with the onset of synaptic function, but to proceed slowly so that even at 6 months the myelin coat is still thinner than normal ${ }^{16,31}$. In addition, a substantial fraction of the optic fibers, primarily those of smaller diameter, remain unmyelinated for long periods. This uneveness in the process of myelination probably causes the formation of the double peaked responses seen at 84 days (Fig. 2). Most but not all of the regenerated but unmyelinated optic fibers seemed to be gone by 161 days.

\section{The effect of TTX during regeneration}

The only significant difference between the TTX fish and the controls was the longer latency seen in the 28-31 day group. At this point when fibers are still unmyelinated, this is likely to reflect a smaller average diameter for the axons in the TTX fish. This is consistent with a $60 \%$ deficit in the amount of axonally transported material reaching the tectum at 21-28 days as found by Edwards and Grafstein ${ }^{6}$. Both the deficit in transport and the difference in latency disappear within two weeks thereafter. Moreover, that study ${ }^{6}$ showed that although there were no differences in the size or number of retinal ganglion cells size or number and although the leading axons seemed to reach the tectum at approximately the same time, there was a reduction in the size or number of regenerating axons in the optic nerves of the TTX fish. Any retardation in axonal outgrowth might be expected to produce a smaller field potential at 28-31 days. The mean response amplitude for the TTX fish was $8 \%$ smaller, but this difference was not significant. Apparently the variability of the regeneration process overwhelms any small difference that might exist between TTX and control fish.

Edwards and Grafstein ${ }^{6}$ also tested for the return of various behaviors (startle response, food pellet localization, dorsal light reflex) in TTX blocked fish, and found that the return was delayed by $4-8$ days relative to control fish injected with citrate. Both the startle reaction and the dorsal light reflex do not depend upon retinal connections to the tectum ${ }^{27}$, and the delay in their return can be largely attributed to the retardation of axonal outgrowth caused by TTX' In fact, both this retardation of axonal outgrowth and the delayed return of the startle reaction can be produced by a single injection of TTX at the time of the optic nerve crush. Since the block from a single injection was over long before synapses were formed even in the nontectal areas, there could be no direct effect on synaptogenesis but only an indirect effect through the retardation of axonal elongation.

The delay in the return of food pellet localization $^{6}$, which is a tectally mediated behavior ${ }^{27}$, has no direct correlate in the recordings of field potentials reported here. The amplitude of the potentials in the TTX fish averaged $8 \%$ less than controls, but this difference was not significant. The only reliable difference was the longer latency at 28-31 days, but that is difficult to relate to the return of food pellet localization which normally occurs a week later. It is likely that the delay in the return of this behavior reflects a second effect of TTX on regeneration, an effect upon the sharpening of the retinotopic order as demonstrated in the accompanying paper ${ }^{26}$.

\section{ACKNOWLEDGEMENTS}

We thank Dr. Bernice Grafstein for intiating the collaboration, and Drs. Stephen Easter, David Tieman, Rod Murphey and Suzannah Tieman for useful discussions. This work was supported by NIH Grants EY03736 to J.T.S., NS 09015 and EY 02696 to B. Grafstein and DFG Stu 112/1-1 to C.S. J.T.S. is an Alfred P. Sloan Foundation Fellow. 


\section{REFERENCES}

1 Bergey, G. K., Fitzgerald, S. C., Schrier, B. K. and Nelson, P. G., Neuronal maturation in mammalian cell culture is dependent on spontaneous electrical activity, Brain Research, 207 (1981) 49-58.

2 Brown, M. C. and Ironton, R., Motor neurone sprouting induced by prolonged tetrodotoxin block of nerve action potentials, Nature (Lond), 265 (1977) 459-461.

3 Chung, S. H., Bliss, T. V. P. and Keating, M. J., The synaptic organization of optic afferents in the amphibian tectum, Proc. roy. Soc. Lond. B, 187 (1974) 421-447.

4 Crain, S. M., Bornstein, M. B. and Peterson, E. R., Maturation of cultured embryonic CNS tissues during chronic exposure to agents which prevent bioelectric activity, Brain Research, 8 (1968) 363-372.

5 Crossland, W. J., Cowan, W. M. and Rogers, L. A., Studies on the development of the chick optic tectum. IV. Autoradiographic studies on the retinotectal connections, Brain Research, (1975) 1-23.

6 Edwards, D. L. and Grafstein, B., Intraocular tetrodotoxin in goldfish hinders optic nerve regeneration, Brain Research, 269 (1983) 1-14.

7 Francis A. and Schechter, N., Activity of choline acetyltransferase and acetylcholinesterase in the goldfish tectum after disconnection, Neurochem. Res., 4 (1979) $547-556$.

8 Fujisawa, H., Tani, N., Watanabe, K. and Ibata, Y., Branching of regenerating retinal axons and preferential selection of appropriate branches for specific neuronal connection in the newt, Develop. Biol., 90 (1982) 43-57.

9 Harris, W. A., The effects of eliminating impulse activity on the development of the retinotectal projection in salamanders, J. comp. Neurol., 194 (1980) 303-317.

10 Hodgkin, A. L. and Huxley, A. F., The dual effect of membrane potential on sodium conductance in the giant axon of Loligo, J. Physiol. (Lond.), 116(1952) 497-506.

11 Hubel, D. H., Wiesel, T. N. and LeVav, S., Plasticity of ocular dominance columns in monkey striate cortex, Phil. Trans. B, 278 (1977) 409.

12 Keating, M. I., The time course of experience dependent synaptic switching of visual connections in Xenopus laevis, Proc. roy. Soc. Edinb. B., 189 (1975) 603-610.

13 Leghissa, S., La struttura microscopia e la citoarchitettonica del tetto ottico dei pesci teleostei, $Z$. Anat. Entwl.Gesch., 118 (1955) 427-463.

14 Mesulam, M.-M., Tetramethyl benzidine for horseradish peroxidase neurohistochemistry: a noncarcinogenic blue reaction product with superior sensitivity for visualizing neural afferents and efferents, J. Histochem. Cytochem., $26(1978) 106-117$.

15 Meyer, R. L., Mapping the normal and regenerating retinotectal projection of goldfish with autoradiographic methods, J. comp. Neurol., 189 (1980) 273-289.
16 Murray, M. and Edwards, M. A., A quantitative study of the reinnervation of the goldfish optic tectum following optic nerve crush, J. comp. Neurol., 209 (1982) 363-373.

17 McQuarrie, I. G. and Grafstein, B., Effect of a conditioning lesion on optic nerve regeneration in goldfish, Brain Research, 216 (1981) 253-264.

18 Norden, J. J. and Freeman, J. A., Quantitative ultrastructural analysis of the goldfish tectum following enucleation, Soc. Neurosci. Abstr., 6(1980) 293.

19 Obata K., Development of neuromuscular transmission in culture with a variety of neurons and in the presence of cholinergic substances and tetrodotoxin, Brain Research, 119 (1977) 141-153.

20 Oswald, R. E., Schmidt, J. T., Norden, J. J. and Freeman, J. A., Localization of bungarotoxin binding sites to the goldfish retinotectal projection, Brain Research, 187 (1980) 113-128.

21 Oswald, R. E. and Freeman, J. A., Characterization of the nicotinic acetylcholine receptor isolated from goldfish brain, J. Biol. Chem., 254 (1979) 3419-3426.

22 Pestronk, A. and Drachman, D. B., Motor nerve sprouting and acetylcholine receptors, Science, 199 (1978) 1223-1225.

23 Schechter, N., Francis, A., Deutsch, D. G. and Gazzaniga, M. S., Recovery of tectal nicotine-cholinergic receptor sites during optic nerve regeneration in goldfish, Brain Research, 166 (1979) 57-64.

24 Schmidt, J. T., The laminar organization of optic nerve fibers in the tectum of goldfish, Proc. roy. Soc. Lond. B., 205 (1979) 287-306.

25 Schmidt, J. T. and Freeman, J. A., Electrophysiologic evidence that the retinotectal projection in the goldfish is nicotinic cholinergic, Brain Research, 187 (1980) 129 142.

26 Schmidt, J. T. and Edwards, D. L., Activity sharpens the map during the regeneration of the optic nerve in goldfish, Brain Research, 269 (1983) 29-39.

27 Springer, A. D. and Agranoff, B. W., Effect of temperature on rate of goldfish nerve regeneration: a radioautographic and behavioral study, Brain Research, 128 (1977) $405-415$.

28 Stuermer, C. Easter, S. S., Regenerating optic fibers of goldfish do not follow their old pathways in tectum, A.R.V.O., in press.

29 Stuermer, C. L., Regenerating optic axons in the godfish: spatiotemporal pattern of synapse formation. Invest. Ophthal. visual Sci., 20 Suppl. 176 and manuscript in preparation.

30 Stryker, M. P., Late segregation of geniculate afferents to the cat's visual cortex after recovery from binocular impulse blockade, Soc. Neurosci. Abstr., 7(1981) 842.

31 Wolburg, H., Growth and myelination of goldfish optic nerve fibers after retina regeneration and nerve crush, $Z$. Naturforsch, 33 (1978) 988-996. 